\title{
A Retrospective study on the dynamics of the clinical and Demographical evolution of Cutaneous Leishmaniasis in the Yazd province, Iran
}

\author{
Ali Fattahi Bafghi ${ }^{1}$, Elham Rezaee*2 \\ 1 PhD D in Parasitology, Department of medical Parasitology and mycology, Shahid Sadough, University of medical sciences, Yazd, \\ Iran. \\ 2 MSc student in Parasitology Department of medical Parasitology and Mycology, Shahid Sadoughi University of Medical Sciences, \\ Yazd, Iran.
}

*Corresponding Author: Mrs. Elham Rezaee, Parasitology, Department, Paramedical School, Gerash University of Medical

Sciences, Fars province, Gerash, Iran

Email: rezaee.para92@gmail.com, Tel: (071) 52448101, Fax: (071) 52452335.

\begin{abstract}
Leishmaniasis is considered as an important health problem in the world. Cutaneous Leishmaniasis is epidemic in all of places of Iran, this disease begins as small swells and then they gradually grow and turn into wounds. Since the epidemiological study of this problem is effective in preventing and controlling it. The current research was conducted to study the epidemiological study of Cutaneous Leishmaniasis in Yazd province during the past 5(2014-2018) years. This cross-sectional study has been conducted on registered information and documents of the patients having problem in Yazd province remedial and health care centers over the past 5 years. First, the registered demographic and epidemiological data of the patients were extracted and then they were analyzed by using SPSS 18 software with chi-square test and descriptive statistics. 897 patients included 457 males (51.06\%) and 439 females (48.94\%). The majority of the patients were between nine 20-29 years old and the minority of them were over the range of 5-9 years old. Most lesions were on the hand and leg of the patients. It was also found that there was a significant relationship between age groups and types of Leishmaniasis $(P<0.05)$ as well as gender and type Leishmaniasis $(P<0.05)$. According to our study, there has been an ascending trend in disease frequency in Yazd province from 2014 to 2018. This increase in diseases due to lack of health and educational status of the people-is also a lack of personal hygiene.
\end{abstract}

Keywords: Frequency, Cutaneous Leishmaniasis, Epidemiology, Yazd.

\section{INTRODUCTION}

Leishmaniasis is an infectious disease that the World Health Organization estimates, $10 \%$ of the world's population are at risk for Leishmaniasis. Prevalence of disease is 12 million people and its incidence is 2 to 1.5 million. Disease has been reported from more than 98 countries, mostly developing countries (77 countries). The Cutaneous form is more common than other types (visceral and mucous -cutaneous), so that each year nearly 70 percent of all reported new cases of is Cutaneous Leishmaniasis. $90 \%$ of cases of Cutaneous Leishmaniasis are in Iran, Afghanistan, Saudi Arabia, Syria, Brazil and Peru. Leishmaniasis is a disease transmitted by arthropods, which is one of the major health problems in the world, especially in Iran [2]. Annually more than 20,000 new cases reported in Iran. Leishmaniasisis in 17 endemic provinces, including Isfahan, Yazd, Khorasan Razavi, Kerman, Ilam, Fars and Golestan [3-4]. Cutaneous Leishmaniasis caused by a protozoan called Leishmaniathe Kinotoplastida class that in terms of their living environment can be seen in two forms without flagella (amastigotes) and flagellate (promastigotes). Host species of this disease is insects called sand flies of the genus Phlebotomus. Cutaneous leishmaniasisare classified as in Anthroponotic (dry) and Zoonotic (wet) forms, Leishmania major and Leishmania tropica are causes of them respectively [5] Clinical form of Cutaneous Leishmaniasis can include centered auto- limiting skin lesions or nodules to the metastasis and 
expanding form.

\section{MATERIALS AND METHODS}

This descriptive-analytical study was conducted on the Cutaneous Leishmaniasis victims who had visited health centers of Yazd since the beginning of April 2014 to the end of March 2018. The required information such as the patient's national origin, age, sex, duration of disease, illness, duration of treatment, the diagnosis, geographic region, scars, patient's job and the city or village where the patient has traveled over the past year were extracted and analyzed. Moreover, the patients' demographic data were analyzed via SPSS softwareandusing Chi-square test. Information required includes to the extraction and analysis of was. Patients' demographic data with SPSS software using Chi-square test was performed.

\section{RESULTS}

The information of 897 victims with Cutaneous Leishmaniasis who were treated in health centers of Yazdsince the beginning of April 2014 to March 2018, were collected. According to obtained information, frequency of both types of Cutaneous
Leishmaniasis based on the year is presented in Figure 1, which, according to 5-year-old study, the highest incidence of Cutaneous Leishmaniasis was in 2014 and the lowest was 2018. The highest rates of infection were in autumn and the lowest was in winter (Figure 1).

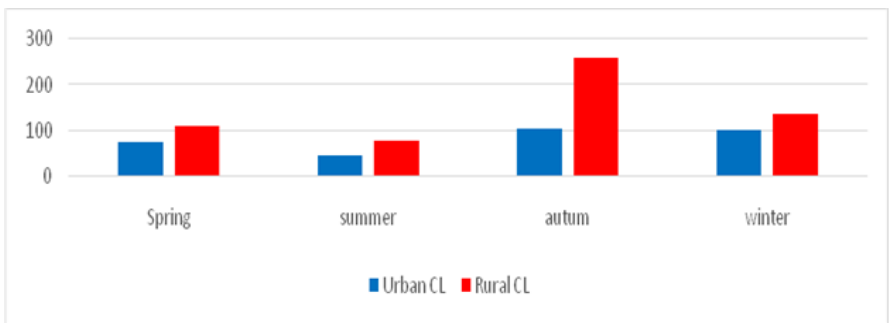

Figure 1: The infection rate to Cutaneous Leishmaniasis based on season by 2014- 2018 years.

The demographic data of patients with Cutaneous Leishmaniasis showed according to Year, Season, Gender, Age and Nationality by $2014-2018$ years (Table 1 ).

Table 1: The infection rate to Cutaneous Leishmaniasis based on Year, Season, Gender, Age and Nationality by 2014-2018 years.

\begin{tabular}{|c|c|c|c|c|c|}
\hline \multicolumn{2}{|l|}{ Variables } & \multicolumn{2}{|c|}{ Cutaneous Leishmaniasis } & \multirow{2}{*}{$\begin{array}{l}\text { Total } \\
139(100 \%)\end{array}$} & \multirow{2}{*}{$\begin{array}{l}\text { P-value } \\
<0.001\end{array}$} \\
\hline Year & 2014 & $\begin{array}{l}\text { Urban CL } \\
107(77 \%)\end{array}$ & $\begin{array}{l}\text { Rural CL } \\
32(23 \%)\end{array}$ & & \\
\hline & 2015 & $137(77.8 \%)$ & $39(22.2 \%)$ & $176(100 \%)$ & \\
\hline & 2016 & $110(57.3 \%)$ & $82(42.7 \%)$ & $192(100 \%)$ & \\
\hline & 2017 & $146(62.9 \%)$ & $86(37.1 \%)$ & $232(100 \%)$ & \\
\hline & 2018 & $75(47.5 \%)$ & $83(52.5 \%)$ & $158(100 \%)$ & \\
\hline \multirow[t]{4}{*}{ Season } & Spring & $108(59.3 \%)$ & $74(40.7 \%)$ & $182(100 \%)$ & \multirow[t]{4}{*}{0.001} \\
\hline & Summer & $75(62.5 \%)$ & $45(37.5 \%)$ & $120(100 \%)$ & \\
\hline & Autumn & $120(33.34 \%)$ & $220(66.66 \%)$ & $360(100 \%)$ & \\
\hline & Winter & $105(44.68 \%)$ & $130(55.32 \%)$ & $235(100 \%)$ & \\
\hline \multirow[t]{2}{*}{ Gender } & Male & $314(68.7 \%)$ & $143(31.3 \%)$ & $457(100 \%)$ & \multirow[t]{2}{*}{0.003} \\
\hline & Female & $200(68.34 \%)$ & $139(31.66 \%)$ & $439(100 \%)$ & \\
\hline \multirow[t]{5}{*}{ Age } & $<15$ & & & $175(100 \%)$ & \multirow[t]{5}{*}{0.072} \\
\hline & $15-30$ & & & $262(100 \%)$ & \\
\hline & $30-45$ & & & $186(100 \%)$ & \\
\hline & $45-60$ & & & $163(100 \%)$ & \\
\hline & $>60$ & & & $111(100 \%)$ & \\
\hline \multirow[t]{2}{*}{ Nationality } & & & & $778(100 \%)$ & \\
\hline & & & & $119(100 \%)$ & \\
\hline
\end{tabular}


The highest rates of infection were in 2010 and the lowest was in 2014(Figure 2).

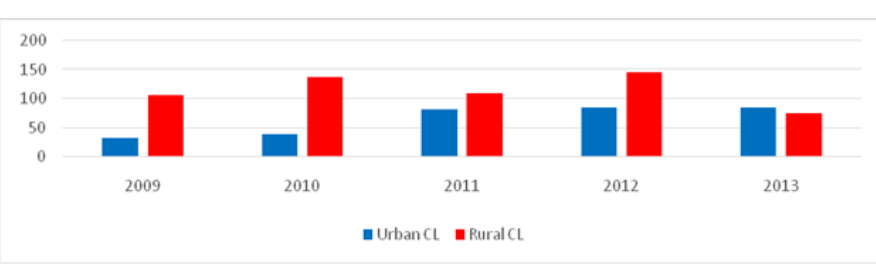

Figure 2: The infection rate to Cutaneous Leishmaniasis based on the type of disease by 2009-2018 years.

According to the results, most lesions were in the hands and feet $(83.1 \%)$ and the head and face $(9-10 \%)$ and both $(6 \%)$ respectively (Table 2 ).

Table 2: The frequency and percentage of siteof lesions

\begin{tabular}{|l|l|l|}
\hline \multirow{2}{*}{ Site Of Lesion } & \multicolumn{2}{|c|}{ Distribution } \\
\cline { 2 - 3 } & Frequency & Percentage \\
\hline Head and Face & 98 & 10.9 \\
\hline Hands and feet & 745 & 83.1 \\
\hline both & 54 & 6.0 \\
\hline
\end{tabular}

The results revealed that Glucantime drug is the most used therapeutic protocol for victims in Yazd (Figure 3).

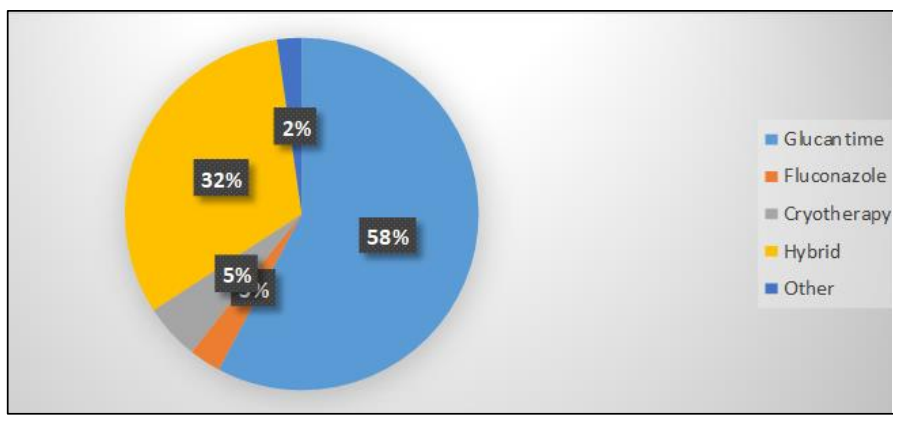

Figure 3: Percentage of the different therapeutic methods.

Table 3: Frequency distribution of Cutaneous Leishmaniasis based on geographic area

\begin{tabular}{|l|l|l|l|l|}
\hline \multirow{2}{*}{ Geographic Area } & \multicolumn{4}{|l|}{ Type of Leishmaniasis } \\
\cline { 2 - 5 } & \multicolumn{2}{|l|}{ Zoonotic } & \multicolumn{2}{l|}{ Anthroponotic } \\
\cline { 2 - 5 } & No & Percentage & No & Percentage \\
\hline Center & 113 & 67.3 & 55 & 32.7 \\
\hline East & 73 & 59.8 & 49 & 40.2 \\
\hline West & 39 & 76.5 & 12 & 23.5 \\
\hline South & 56 & 68.3 & 26 & 31.7 \\
\hline North & 294 & 62.0 & 180 & 38.0 \\
\hline Total & 575 & 64.1 & 322 & 35.9 \\
\hline
\end{tabular}

\section{DISCUSION}

It has utmost importance, since epidemiological and ecological studies that examined risk factors in the spread of disease, as a result, the make more effective the control and prevention activities. Since in controlling the diseases transmitted by insects, comprehensive epidemiological and ecological information about their disease, geographical distribution, seasonal abundance and their association with factors such as age, sex, history of travel to endemic areas, etc., must be collected. It should be noted that without this information in one area cannot be accomplished policies and control measures. According to location and climate of Yazd city that is susceptible to an outbreak of Cutaneous Leishmaniasis, which every year a significant number of these people referred to health centers of Yazd city. The main aim of this study was to review Cutaneous Leishmaniasis in the city of Yazd. Study of Demographic characteristics such as age, sex, nationalityare other objectives of the study. Among 897cases referred to health centers in Yazd during the years 2014 till 2018 that suspected to Cutaneous Leishmaniasis, affection of 896 persons to Cutaneous Leishmaniasis was confirmed. The results revealed that, due to ecological situation of city, the prevalence of disease in Yazd is high. Despite modernization in different urban areas, because of the old and non-residential houses in these areas can be place of growth and living rodents that can play the tank role. The results revealed that the most cases of Cutaneous Leishmaniasis is related to north of the city of Yazd, which mentioned region have the old context and wasteland.

Tashrifi et al. in the review of all patients with suspicious lesions to health center in the first seven months of 1390 in Esfarāyen found, on 154 cases of Cutaneous Leishmaniasis in 1390 sexually among which84 (54\%) were female and 70 (46\%) were men and in terms of location 130 (95\%) of them in the village, $24(15 \%)$ of them dwelt in the city.among which 7 relapsed cases $(4.5 \%)$ in the meantime, and 146 patients (94\%) new cases and one case $(0.6 \%)$ were treatment failures. In only 18 cases $(11.6 \%)$ co-infection with other family members was reported. In terms of age distribution of the patients, $76(49 \%)$ were under 10 years, $22(14.2 \%)$ between 10 to 20 years and $16(10.3 \%)$ between 20 to 30 years and $18(11.6 \%)$ between 30 to 40 years and 11 (7.1\%) between 40 to 50 years, 11 patients $(7.1 \%)$ were above 50 years. In regard withoccupation, $41.5 \%$ were children, $14.2 \%$ were housewives, $2.5 \%$ were rancher, 19.4 were students, $1.9 \%$ was farmers and $9 \%$ were in other professions. $149(97.5 \%)$ have Zoonotic Cutaneous Leishmaniasis and $4(2.5 \%)$ have Anthroponotic Cutaneous Leishmaniasis altogether [6-8]. In Jamshidi et al. study in 1384 with a review of Cutaneous Leishmaniasis in rural Abarkouh, 20 villages of Abarkouh city with a population of over 15515 people and 3456 households with cluster sampling, 20 clusters that each cluster has 15, contains 300 households were selected for review then questionnaires were completed after examination of households.

\section{CONCLUSION}

Based on results of this study, the prevalence of rural Zoonotic Cutaneous Leishmaniasis in Yazd is more than urban Cutaneous Leishmaniasis and also due to the increasing prevalence of urban Cutaneous Leishmaniasis over years 2014 to 2018 , this requires measures to prevent and fight against the vector and reservoir.

\section{Conflict of Interest}

We declare that we have no conflict of interest.

\section{Financial Support}

None declared. 


\section{REFERENCES}

1. World Health Organization. WHO report on global surveillance of epidemic-prone infectious diseases, 2000.

2. Rafati S, Modabber F. Cutaneous Leishmaniasis in Middle East and North Africa. PLoS negl trop dis. 2014; 117-39.

3. Yaghoobi-Ershadi MR, Marvi-Moghadam N, Jafari R, Akhavan AA, Solimani H, Zahrai-Ramazani AR, et al. Some epidemiological aspects of cutaneous Leishmaniasis in a new focus, Central Iran. Dermatol Res Pract. 2015. Article ID 286408, 5 pages, 2015. doi:10.1155/2015/286408.

4. Feiz-Haddad $\mathrm{MH}$, Kassiri $\mathrm{H}$, Kasiri $\mathrm{N}$, Panahandeh A, Lotfi $\mathrm{M}$. Prevalence and epidemiologic profile of acute cutaneous Leishmaniasis in an endemic focus, Southwestern Iran. Journal of Acute Disease. 2015, Article in press.

5. Leprohon P, Fernandez-Prada C, Gazanion É, Monte-Neto R, Ouellette M. Drug resistance analysis by next generation sequencing in Leishmania. Int J Parasitol Drugs Drug Resist. 2015; 5(1):26-35.

6. Ali Fattahi Bafghi, Ali Reza Vahidi, Mohammad Hossein Anvari, Kazem Barzegar, Mahin Ghafourzadeh, The in vivo Antileishmanial activity of alcoholic extract from Nigella sativa seeds, African Journal of Microbiology Research. 2011; 5(12):1504-1510.

7. Ali Fattahi Bafghi, Mohammad Noorbala, Mohammad Taghi Noorbala, Mahdi Aghabagheri. Anti Leishmania Effect of Zinc Sulphate on the Viability of Leishmania tropica and L. major Promastigotes, Jundishapur J Microbiol. 2014; 7(9): e11192.

8. Kerimyan Shirazi M, Razmi G, Naghibi A. Evaluation of the patients referred to the laboratory for diagnosis of Cutaneous Leishmaniasis in Mashhad. Jornal of Reserch and health. 2015; 5(1):121[Persian]

9. Ali Fattahi Bafghi, Sayyed Majid Bagheri, Sayyed Hassan Hejazian, 2014. Antileishmanial activity of Ferula assa-foetida oleo gum resin (asafetida) against Leishmania major: An in vitro study, Journal of Ayurveda \& Integrative Medicine, 2014

10. Hamzavi, Yazdan, Nahid Khademi. "The Analytical study of Cutaneous Leishmaniasis in Kermanshah, 2011.

11. Ali Fattahi Bafghi, Mojtaba Daghighi, Karim Daliri, Ali Jebali, Magnesium oxide nanoparticles coated with glucose can silence important genes of Leishmania major at sub-toxic concentrations. Colloids and Surfaces B: Biointerfaces, 2015; 136(2015):300-304.

12. Tohidi, Faride, Afsane Barghae. "Cutaneous Leishmaniasis Parasite Identification via PCR in the Infected Areas in Golestan Province." 2011; 26-31[Persian].

13. Jamshid Ayatollahi, Mehran Karimi, The prevalence of cutaneous Leishmaniasis in rural Abarkuh, Yazd province, Iran, Vlo. 10, No. 30, 2005, Quarterly tropical and Infectious diseases of Iran, 2011.

14. Ali Fattahi Bafghi, Ali Jebali, Karim Daliri, Silica nanowire conjugated with loop-shaped oligonucleotides: A newstructure to silence cysteine proteinase gene in Leishmania. Colloids and Surfaces B: Biointerfaces, 2016; 136:323-328.

15. Ali Fattahi Bafghi, Mahmood Dehghani Ashkezarib,, Mahmoud Vakili, Soheila Pournasir. 2018. Polyurethane sheet impregnated with Arabinogalactan can lead to increase of attachment of promastigotes and Amastigote of Leishmania major (MRHO/IR/75/ER) by GP63 and HSP70 genes, Materials Science \& Engineering C, 292-296.

16. Epidemiological study in \& new focus of cutaneous Leishmaniasis due to major in Ardestan town, central Iran- Asia Trop 2001; 79(2):115-21 